\title{
Preço de transferência em empresas exportadoras brasileiras: adoção para avaliação de
}

\section{desempenho financeiro de divisões}

Joice Denise Schäfer

Doutoranda em Administração-Universidade Federal de Santa Catarina

schafer.joice@gmail.com

https://orcid.org/0000-0001-6779-1079

Valdirene Gasparetto

Professora do Programa de Pós-Graduação em Contabilidade da Universidade Federal de Santa Catarina

valdirenegasparetto@gmail.com

http://orcid.org/0000-0002-2825-4067

Luiz Felipe Ferreira

Controlador Geral do Estado de Santa Catarina. Professor do Programa de Pós-Graduação em Contabilidade

Universidade Federal de Santa Catarina

luiz.felipe@ufsc.br

https://orcid.org/0000-0002-6162-8905

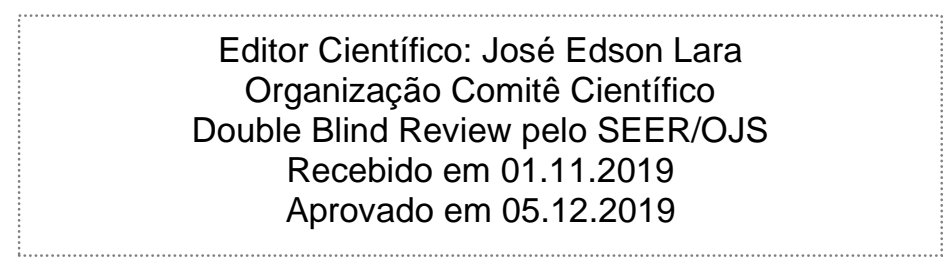

Este trabalho foi licenciado com uma Licença Creative Commons - Atribuição - Não Comercial 3.0 Brasil 


\title{
Resumo
}

Objetivo do estudo: Este estudo tem o objetivo de verificar fatores contingenciais que levam empresas exportadoras brasileiras a utilizar preço de transferência com abordagem gerencial.

Metodologia: Pesquisa de levantamento foi realizada com noventa empresas exportadoras brasileiras, sendo aplicada aos dados a análise fatorial e a regressão logística.

Originalidade: As diferenças entre os métodos de preço de transferência são discutidas com frequência na literatura, principalmente, por meio de modelos econométricos e quaseexperimentos. Por outro lado, pouco vem se discutindo sobre a adoção desta ferramenta gerencial na prática das organizações e sobre os fatores que influenciam o seu uso.

Principais resultados: Os resultados mostram que, para a amostra, os fatores contingenciais investigados - descentralização, tecnologia, estratégia, tamanho da empresa e estabilidade do ambiente - não apresentaram relação significativa com o uso de preço de transferência, revelando que, mesmo empresas que adotam, normalmente empregam métodos que não permitem avaliação do desempenho financeiro das divisões.

Contribuições teóricas: Apesar da relevância do preço de transferência, mencionada pela literatura pesquisada, ele é pouco empregado na prática das organizações brasileiras pesquisadas. Isso indica a preferências da alta gestão pelo uso de indicadores não financeiros para a avaliação do resultado das unidades descentralizadas, ou ainda, a falta de conhecimento dos profissionais gerenciais acerca do preço de transferência aplicado para esta finalidade.

Palavras-chave: Preço de transferência gerencial; preço interno; fatores contingenciais; empresas exportadoras; avaliação de desempenho.

\section{Transfer price in Brazilian exporting companies: adoption for division's financial}

\section{performance evaluation}

\begin{abstract}
Purpose: This study aims to verify contingency factors that lead Brazilian exporting companies to use transfer pricing with a management approach.

Design: This survey was applied to ninety Brazilian exporting companies. Factorial analysis and logistic regression were used for data analysis.

Originality: Differences between transfer pricing methods are often discussed in the literature, mainly through econometric models and quasi-experiments. On the other hand, little has been discussed about the adoption of this management tool in the practice of organizations and about the factors that influence its use.

Findings: The results show that, for the sample, the contingency factors investigated decentralization, technology, strategy, company size and environmental stability - did not present a significant relation with the use of transfer pricing, revealing that Even companies that apply the internal transfer price use methods that do not allow the assessment of the financial performance of divisions.

Theoretical contributions: Despite the relevance of transfer pricing, mentioned in the researched literature, it is little used in the practice of the Brazilian organizations researched. This indicates the preferences of top management for the use of non-financial indicators to evaluate the results of decentralized units, or the lack of knowledge of management professionals about the transfer price applied for this purpose.
\end{abstract}


Keywords: Managerial transfer price; internal price; contingency factors; exporting companies; performance evaluation.

\section{Precio de transferencia en empresas exportadoras Brasileñas: adoção para evaluación de rendimiento financiero de divisiones}

\section{Resumen}

Propósito del estúdio: Este estudio tiene el objetivo de verificar factores contingentes que llevan a empresas exportadoras brasileñas a utilizar precio de transferencia con abordaje gerencial.

Metodología: La encuesta fue realizada con noventa empresas exportadoras brasileñas, siendo aplicada a los datos el análisis factorial y la regresión logística.

Originalidad: Las diferencias entre los métodos de precios de transferencia a menudo se discuten en la literatura, principalmente a través de modelos econométricos y cuasiexperimentos. Por otro lado, poco se ha discutido sobre la adopción de esta herramienta de gestión en la práctica de las organizaciones y sobre los factores que influyen en su uso.

Resultados: Los resultados muestran que, para la muestra, los factores contingentes investigados - descentralización, tecnología, estrategia, tamaño de la empresa y estabilidad del ambiente - no presentaron relación significativa con el uso de precio de transferencia, revelando que, incluso empresas que adopta, normalmente emplean métodos que no permiten evaluar el rendimiento financiero de las divisiones.

Contribuciones teóricas: A pesar de la relevancia del precio de transferencia, mencionado en la literatura investigada, es poco utilizado en la práctica de las organizaciones brasileñas encuestadas. Esto indica las preferencias de la alta gerencia para el uso de indicadores no financieros para evaluar los resultados de las unidades descentralizadas, o la falta de conocimiento de los profesionales de la gerencia sobre el precio de transferencia aplicado para este propósito.

Palabras clave: Precio de transferencia gerencial; precio interno; factores contingenciales; empresas exportadoras; evaluación del desempeño.

\section{Introdução}

Preço de transferência é um instrumento contábil que as empresas podem empregar com foco fiscal ou gerencial. Na abordagem de preço de transferência gerencial, adotada nesta pesquisa, o foco está no provimento de informações financeiras para avaliação de desempenho de unidades organizacionais descentralizadas (divisões, centros de responsabilidade, áreas ou departamentos), quando a gestão é feita pela organização como se fossem unidades autônomas, normalmente centros de lucro ou de investimentos. Nesse contexto, o preço de transferência refere-se ao preço de um bem ou serviço transferido de uma unidade para outra da mesma 
organização, que influencia o lucro e o ROI de cada divisão, e pode influenciar o resultado da organização como um todo (Baldenius, Reichelstein, \& Sahay, 1999).

Hirshleifer, em 1956, abordava preço de transferência com base na teoria econômica, e indicava o método do custo marginal como solução ótima para a transferência interna de um item quando a unidade vendedora não estivesse operando a plena capacidade e não houvesse um preço disponível para o item no mercado, o que é comum no caso de produtos semiacabados. Não havendo capacidade ociosa, o autor demonstrou que o método do preço de transferência mais adequado era baseado no mercado, e noutras situações seria necessário empregar o preço negociado pelas divisões envolvidas. Desde então, diferentes estudos apontaram acertos (Banker \& Datar, 1992) e dificuldades (Enzer, 1975; Shi, Kwak, \& Lee, 1998; Alles \& Datar, 1998; Göx, 2000; Ruiz-Aliseda, 2003) da proposta de Hirshleifer (1956).

Nos anos mais recentes os estudos têm abordado o uso desses e de outros métodos de preço de transferência, como preço de mercado ajustado, dual price, preço baseado nos custos (considerando diferentes abordagens como custo-padrão, custo total, custo total mais margem, custo variável, entre outros) e preço administrado (Borkowski, 1992; 1997; Grunow, Beuren, \& Hein, 2010; Johnson, Johnson, \& Pfeiffer, 2016) e também avaliando o efeito da precificação interna sobre os resultados da empresa e a autonomia e motivação dos gestores (Baldenius, Reichelstein, \& Sahay, 1999; Wolff, 2007; Johnson et al., 2016).

Dentre os estudos há investigações empíricas, abordando como os métodos são empregados nas empresas e seus efeitos sobre as divisões, gestores e empresa como um todo (Bailey \& Collins, 2005; Arya \& Mittendorf, 2007; Adams \& Drtina, 2008; Cheng \& Hsieh, 2009) e os fatores que levam as organizações a optarem por determinado método de precificação interna. Esses estudos são principalmente internacionais. No Brasil, cita-se o estudo de Grunow et al. (2010), que identificaram os métodos de preço de transferência interna utilizados nas maiores empresas do Brasil. Em uma amostra de 38 empresas os autores identificaram que predominava o método de preço com base no custo, método este que não se aplica à avaliação do desempenho dos centros de responsabilidade.

Os estudos sobre preço de transferência normalmente trazem discussões normativas e empíricas sobre os métodos de preço de transferência que são, ou deveriam ser, usados por empresas com diferentes características. A preocupação sobre a adoção ou não, de preço de transferência, não tem sido abordado com frequência nos estudos, havendo evidências de que a literatura sobre preço de transferência avançou na discussão dos métodos, sem analisar o que determina o seu uso pelas organizações. 
A investigação dos fatores que levam as organizações a optarem por determinado método de precificação interna, partem, em sua maioria, do pressuposto de que fatores ambientais e organizacionais, tais como grau de descentralização (Borkowski, 1990, 1992; Holmstrom \& Tirole, 1991; Kouser et al., 2012),, tecnologia (Adler, 1996; Dikolli \& Vaysman, 2006), estratégia de diversificação e integração da empresa (Eccles, 1985; Spicer, 1988; Borkowski, 1990; Adler, 1996; Kouser et al., 2012), tamanho da empresa (Borkowski, 1990, 1992, 1996; Kouser et al., 2012), além de fatores externos à organização como ameaças, incerteza e estabilidade do ambiente (Borkowski, 1990, 1992), dentre outros (Mahenthiran, Greenberg, \& Greenberg, 1993; Shih, 1996; Kachelmeier \& Towry, 2002; Ghosh, 2006; Chang et al., 2008; Bhattacharjee \& Moreno, 2017), influenciam na definição do método de preço de transferência, entretanto, não há comprovação de que tais variáveis são de fato determinantes na adoção deste instrumento.

Assim, a partir das características investigadas pela literatura, como determinantes para seleção do método de preço de transferência adotado pelas empresas, este estudo tem o objetivo de verificar os fatores que levam empresas exportadoras brasileiras a utilizar o preço de transferência com abordagem gerencial, para avaliação de desempenho financeiro de divisões. Para isso, além desta seção introdutória, este trabalho apresenta uma seção de referencial sobre as discussões desenvolvidas na literatura sobre preço de transferência e as hipóteses oriundas dessas discussões, posteriormente, a metodologia empregada, a análise e discussão dos resultados e, por fim, as conclusões do estudo.

\section{Fundamentação teórica e hipóteses da pesquisa}

Nas últimas décadas, pesquisas sobre preço de transferência investigaram os métodos empregados pelas empresas, dando ênfase ao método do preço de mercado, que se refere ao preço praticado em operações normais de mercado, com clientes externos, que pode ser também ajustado, reduzindo gastos que a empresa não incorre em transações internas (Borkowski, 1996; Vaysman, 1996; Wolff, 2007); método do preço baseado nos custos, que pode referir-se às empresas que usam apenas o custo variável para valorar suas transferências, ou então custo total, custo total mais uma margem de lucro, custo-padrão, entre outros (Borkowski, 1996; Grunow et al., 2010); método dual price, quando a divisão compradora recebe o bem transferido pelo preço equivalente ao custo, enquanto a divisão vendedora contabiliza a transferência pelo preço de mercado (Eccles, 1983; Johnson et al., 2016); método do preço 
negociado, quando os gerentes das duas unidades - vendedora e compradora - se reúnem para entrar em consenso sobre o preço da transação (Flavell, 1977; Dikolli \& Vaysman, 2006); e método do preço administrado, que se refere à interferência do administrador na transação, impondo uma regra em relação ao preço pelo qual a transferência ocorrerá.

Os estudos sobre preço de transferência abrangem três linhas de estudo: i) identificação do melhor método de preço de transferência a ser utilizado pela empresa - que abrange tanto os métodos tradicionais considerados na literatura, quanto a proposição de modelos econômicos e matemáticos; ii) efeitos que os métodos empregados trazem para as divisões, seus gestores e empresa como um todo; e iii) fatores que influenciam a decisão entre um ou outro método de precificação interna.

Na primeira linha, há estudos que indicam os melhores modelos a serem empregados nas organizações, em que os gestores divisionais possuem maior número de informações do que a alta administração da empresa (Vaysman, 1998), e em casos em que há divergência entre os objetivos de ambos e assimetria informacional (Banker \& Datar, 1992) ou, ainda, que apontam as restrições que podem ser impostas às divisões para que adotem o preço de transferência ideal para os produtos intermediários (Dorestani, 2004) e simulações de diferentes cenários, para identificar o preço de transferência ótimo de acordo com critérios múltiplos e vários níveis de restrição (SHI et al.,1998). Outros estudos comparam os métodos de precificação interna conhecidos na literatura, com o objetivo de determinar qual o melhor método a ser empregado em diferentes cenários (Baldenius et al., 1999; Wolff, 2007; Johnson et al., 2016).

McAulay e Tomkins (1992) criticam o excesso de modelagens econômicas e matemáticas acerca de preço de transferência, uma vez que estes modelos são amplamente racionais, complexos e, na maior parte dos casos, difíceis de serem operacionalizados nas empresas. Para os autores, apesar do aumento da literatura da área, ainda não há explicações para as políticas específicas de preço de transferência empregadas pelas empresas (McAulay \& Tomkins, 1992). Gavious (1999) utilizou modelagem econômica, justamente para evidenciar que um esquema simples de preço de transferência é o ideal.

Pesquisas na segunda linha de investigação - sobre os efeitos que os métodos empregados trazem para as divisões, seus gestores e empresa como um todo - são mais recentes, a partir dos anos 2000. Estes estudos contemplam análises sobre o impacto das decisões de preço de transferência na aquisição e fornecimento de insumos (Bailey \& Collins, 2005; Arya \& Mittendorf, 2007), nas decisões de investimentos de capital (Adams \& Drtina), equidade entre divisões (Cheng \& Hsieh, 2009) e bem-estar social. 
$\mathrm{Na}$ terceira linha de pesquisa, sobre os fatores que influenciam a escolha entre um ou outro método de precificação interna, destacam-se estudos sobre o grau de descentralização (Borkowski, 1990, 1992; Holmstrom \& Tirole, 1991; Kouser et al., 2012), a tecnologia (Adler, 1996; Dikolli e Vaysman, 2006), a estratégia de diversificação e integração da empresa (Eccles, 1985; Spicer, 1988; Borkowski, 1990; Adler, 1996; Kouser et al., 2012), o tamanho da empresa (Borkowski, 1990, 1992, 1996; Kouser et al., 2012), além de fatores externos à organização como ameaças, incerteza, estabilidade do ambiente (Borkowski, 1990, 1992), dentre outros (Mahenthiran et al., 1993; Shih, 1996; Kachelmeier \& Towry, 2002; Ghosh, 2006; Chang et al., 2008; Bhattacharjee \& Moreno, 2016).

Os estudos mencionados partem, em sua maioria, da perspectiva de que as empresas utilizam algum método de preço de transferência, assim, dão ênfase à discussão sobre os métodos empregados e não aos fatores que as levam a adotar o preço de transferência. Em especial, a terceira linha de pesquisa busca relações entre variáveis e métodos de preço de transferência, sem que haja uma comprovação anterior da relação entre o uso do preço de transferência e tais características organizacionais e ambientais. Assim, a presente pesquisa busca identificar se as suposições teóricas das quais partem muitas pesquisas em preço de transferência, de fato correspondem à realidade de empresas brasileiras. As variáveis selecionadas e as hipóteses a serem testadas estão apresentadas na sequência.

\subsection{Preço de transferência e descentralização}

A descentralização é considerada um dos pressupostos iniciais para a implementação do preço de transferência nas empresas. A tomada de decisão descentralizada permite que questões inesperadas sejam resolvidas de forma mais rápida e flexível, uma vez que os gestores das unidades tendem a estar mais próximos das informações, o que leva à detecção de problemas e soluções (Liu, Zhang \& Tang, 2015; Johnson et al., 2016).

O preço de transferência permite uma forma de controle central das divisões descentralizadas, sem remover as responsabilidades delegadas. Funciona como um ponto de contato entre as divisões e a direção central, determinando os preços pelos quais os bens e serviços são transferidos (Flavell, 1977). Com a utilização do preço de transferência, as receitas arrecadadas por meio do esforço conjunto das divisões são distribuídas entre elas, gerando uma base para que a empresa possa medir o desempenho financeiro de cada uma das unidades e de seus gestores, que, por sua vez, passam a sentir-se mais motivados diante da perspectiva de uma 
avaliação de desempenho e, em alguns casos, de uma remuneração mais justa (Garrison \& Noreen, 2001). Considerando tais benefícios do preço de transferência para empresas descentralizadas, formulou-se a primeira hipótese deste estudo:

H1 - Há relação positiva entre o nível de descentralização da empresa e o uso do preço de transferência.

Borkowski (1990, 1992), Holmstrom e Tirole (1991) e Kouser et al. (2012) estudaram as relações entre descentralização e métodos de preço de transferência utilizados pelas empresas. Borkowski (1990, 1992) investigou 168 empresas norte-americanas num primeiro estudo e ampliou a amostra em 79 organizações para um segundo, e encontrou relação entre as empresas mais descentralizadas e o uso do preço de transferência negociado. Constatação similar sobre o nível de descentralização e as negociações entre divisões também foi feita por Holmstrome Tirole (1991). Kouser et al. (2012) pesquisaram a existência de relação entre as variáveis descentralização e métodos de preço de transferência - em 43 empresas paquistanesas, mas os resultados não apresentaram significância.

\subsection{Preço de transferência e tecnologia}

De acordo com Adler (1996), algumas dimensões estratégicas devem ser consideradas na definição do método de preço de transferência a ser empregado pelas empresas, entre elas a tecnologia usada para a fabricação do item a ser transferido. $\mathrm{O}$ autor destaca que o investimento em tecnologia pode aumentar os custos das divisões, logo o preço de transferência dos bens e serviços. Caso este investimento não seja feito, no entanto, evitando custos de curto prazo, a empresa tende a perder vantagem competitiva. Assim, empresas que apresentam alto nível tecnológico podem optar por não empregar o preço de transferência, para evitar que os gestores divisionais deixem de investir em melhorias, para reduzir custos e apresentar bons resultados a curto prazo. Diante de tal contexto foi elaborada a segunda hipótese da pesquisa:

H2: Há relação negativa entre o nível tecnológico da empresa e o uso do preço de transferência.

Dikolli e Vaysman (2006) buscaram compreender a relação entre uso da tecnologia, em termos de sistemas de informação e preço de transferência. Os resultados mostram que o ambiente informacional de uma empresa é um fator chave para determinar o método de preço de transferência, e quanto mais restritas as informações (menos tecnologia), maior a tendência das empresas utilizarem o preço negociado, no entanto, à medida que a tecnologia aumenta, o 
custo de oportunidade dos métodos baseados nos custos torna-se melhor. Chenhall (2003), entretanto, destaca que a tecnologia se refere a todos os itens, materiais ou não, necessários para transformar os recursos organizacionais em produtos ou serviços, e não apenas a sistemas de informação, como na abordagem de Dikolli e Vaysman (2006).

\subsection{Preço de transferência e estratégia}

Para Eccles (1983), a estratégia é chave para definição do preço de transferência, havendo duas grandes dimensões estratégicas aplicadas às empresas, a integração vertical e a diversificação. Integração vertical refere-se a uma estratégia organizacional que substitui, completa ou parcialmente, as transações com fornecedores ou distribuidores (Porter, 1996). Diversificação diz respeito ao envolvimento da empresa em diferentes negócios (Eccles, 1983). Eccles (1983) afirma que o nível de adoção destas dimensões estratégicas definirá se a empresa é coletiva, cooperativa, colaborativa ou competitiva, conforme demonstrado na Figura 1.

\section{Figura 1}

Tipos de empresa propostas por Eccles (1983) a partir das dimensões estratégicas

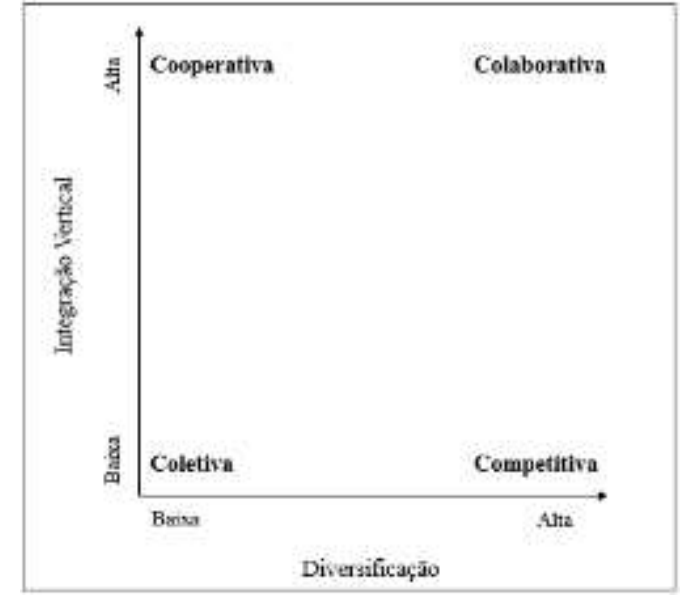

Fonte: Adaptado de Eccles (1983).

Uma empresa com baixa integração e diversificação é classificada como coletiva, e conforme Eccles (1983), tende a não utilizar preço de transferência, pois não possui processos sequenciais ou desenvolvimento de mais de um produto ou serviço. Caso a empresa apresente alta integração vertical, mas não seja diversificada, será classificada como cooperativa, e nesse caso, os centros de responsabilidade normalmente cooperam uns com os outros e o preço de transferência usado tende a ser baseado no custo (real, padrão, ou custo acrescido do investimento). 
Em empresas colaborativas, altamente diversificadas e pouco integradas, as divisões tendem a competir entre si. Nesta situação, o preço com base no mercado ou o dual price são métodos mais recomendados. Nas empresas colaborativas há competição por lucros individuais de cada centro de responsabilidade, entretanto, como os resultados apresentam alta interdependência, é preciso cooperar para melhorar o resultado global (Eccles, 1983).

Spicer (1988) corrobora as ideias de Eccles (1983), de que o preço de transferência é influenciado pela estratégia organizacional em termos de diversificação e integração. Diante das hipóteses levantadas por Eccles (1983) e defendidas por Spicer (1988), de que apenas empresas coletivas - com baixa integração e diversificação - não utilizariam preço de transferência, foi elaborada a terceira hipótese deste estudo:

H3 - Há relação positiva entre a estratégia (integração vertical e diversificação) da empresa e o uso do preço de transferência.

Grau de diversificação e de integração são, portanto, as variáveis utilizadas neste estudo para a análise do fator estratégia. A integração vertical foi analisada anteriormente como direcionador do método de precificação interna por Borkowski (1990) e a diversificação por Kouser et al. (2012). A diversificação também foi destacada por Adler (1996) como uma dimensão estratégica a ser considerada na definição do preço de transferência. Nenhum dos estudos encontrou relação entre as variáveis.

\subsection{Preço de transferência e tamanho da empresa}

De acordo com Chenhall (2003), o aumento do porte das organizações oferece maior oportunidade para expansão das operações, e requer maior controle por meio da utilização de instrumentos gerenciais A influência do tamanho da empresa sobre o uso desses instrumentos foi pesquisada no Brasil por Gonzaga et al. (2010), em 115 empresas no ano de 2007 e 89 empresas no ano de 2008, dentre as listadas entre as 200 maiores do estado do Espírito Santo, e comprovaram a relação entre tamanho da empresa e o uso de benchmarking, orçamento, balanced scorecard, custo padrão, planejamento estratégico, custeio por absorção, custeio variável e preço de transferência. De acordo com os resultados da regressão logística do estudo, as chances de uma empresa com maiores ativos (um desvio padrão a mais) figurar como usuária do preço de transferência é de, aproximadamente, 15\%. Assim como a pesquisa de Gonzaga et al. (2010), a presente pesquisa busca confirmar a relação entre o tamanho da empresa e o uso do preço de transferência. A partir desse contexto propõe-se a quarta hipótese desta pesquisa: 


\section{H4 - Há relação positiva entre o tamanho da empresa e o uso do preço de transferência.}

Os estudos de Borkowski (1990, 1996) também evidenciaram relação entre as variáveis tamanho da empresa e preço de transferência, porém, analisando a prevalência de um método sobre o outro. Borkowski (1990) encontrou que pequenas empresas tendem a utilizar métodos baseados no custo para determinação do preço de transferência, médias empresas optam pelo preço negociado e empresas de grande porte tendem a utilizar mais o preço de mercado. Resultado similar foi encontrado por Borkowski (1996) em uma meta-análise que objetivou avaliar os resultados de estudos de preço de transferência. A autora identificou que que grandes corporações tendem a utilizar métodos baseados no mercado.

As pesquisas de Soutes (2006) e Kouser et al. (2012), por outro lado, não encontraram relação entre as variáveis. Soutes (2006) investigou 90 empresas brasileiras que haviam sido indicadas para o Prêmio Transparência ou estavam na relação de Maiores e Melhores empresas de 2005. Dentre as empresas investigadas, 32\% afirmaram utilizar o preço de transferência com base no mercado e $36 \%$ o preço baseado nos custos. Ao tentar relacionar a adoção desta prática gerencial com o porte da organização, no entanto, não foram encontrados resultados significativos.

\subsection{Preço de transferência e ambiente externo}

Muitas decisões das organizações, com relação aos fatores internos e consequentemente aos instrumentos gerenciais adotados, refletem a influência do ambiente em que a organização está inserida, já que as organizações tendem a adaptar-se ao seu ambiente (Donaldson, 1999). Considerando tais aspectos, Borkowski $(1990,1992)$ investigou a relação entre a estabilidade ambiental e a precificação interna e encontrou evidências significativas de relação entre essas variáveis. Constatou que quanto mais instável o ambiente em que a empresa está situada, mais complexa a tomada de decisão, ou seja, as empresas precisarão de maiores controles, que permitam à alta gestão acompanhar o desempenho de cada divisão, a fim de avaliar a viabilidade de sua manutenção, bem como as compras e vendas de produtos intermediários em mercado externo, e de certa forma a decisão entre produzir ou comprar, o que dá base à formulação da quinta hipótese deste estudo:

H5 - Há relação negativa entre a estabilidade do ambiente em que a empresa está situada e o uso do preço de transferência. 
Chan e Lo (2004) e Mura, Emmanuel e Vallascas (2013) analisaram a influência de outras variáveis ambientais na adoção do preço de transferência, entretanto, mais voltadas ao contexto do preço de transferência fiscal, sendo a investigação da influência destas variáveis inclusive mais comum nas pesquisas de cunho tributário.

\section{Procedimentos metodológicos}

Para este estudo foram investigadas 259 empresas cadastradas no Catálogo de Exportadores Brasileiros, a partir de listagem disponibilizada no site da Confederação Nacional das Indústrias (CNI) e que, a partir de contato inicial por e-mail corporativo, disponibilizaram o contato do controller ou gerente financeiro. Estas empresas foram selecionadas, a partir de constatações como a de Cool, Emmanuel e Jorissen (2008), de que aproximadamente $60 \%$ do total das transações internacionais referem-se a transferências e não a operações de compra e venda. Considerando tal afirmação um percentual significativo das empresas exportadoras tende a realizar transferências.

Para a coleta de dados utilizou-se de questionário, encaminhado para os e-mails informados pelas empresas, respondido via formulário do Google Docs, no período de novembro a dezembro de 2015. Do total de empresas contatadas, obteve-se 90 formulários válidos, ou seja, um retorno de aproximadamente $35 \%$ das empresas.

As variáveis utilizadas no questionário para definir os fatores contingenciais investigados, descentralização, estratégia, tecnologia, tamanho e estabilidade ambiental, estão apresentadas no Quadro 1.

\begin{tabular}{|c|c|}
\hline Fator & Variáveis pesquisadas \\
\hline Descentralização & $\begin{array}{l}\text { Grau da descentralização da tomada de decisão dos gestores divisionais, quanto } \\
\text { ao desenvolvimento de novos produtos (Desc1), contratação e demissão de } \\
\text { pessoal (Desc2), seleção de investimentos relevantes (Desc3), verbas } \\
\text { orçamentárias (Desc4), preço de venda (Desc5) e preço de transferência (Desc6) }\end{array}$ \\
\hline Estratégia & Grau de diversificação (Estra1) e grau de verticalização (Estra2) da empresa \\
\hline Tecnologia & $\begin{array}{l}\text { Grau de utilização de sistema integrado de gestão (Tec1), comércio eletrônico } \\
\text { (Tec2), automatização do processo de produção (Tec3), nível tecnológico das } \\
\text { máquinas e equipamentos (Tec4), qualidade dos insumos (Tec5) e } \\
\text { conhecimentos dos funcionários para o desenvolvimento de suas atribuições } \\
\text { (Tec6) }\end{array}$ \\
\hline Tamanho & Faturamento da empresa (Tam1) \\
\hline $\begin{array}{l}\text { Estabilidade } \\
\text { ambiental }\end{array}$ & $\begin{array}{l}\text { Nível de estabilidade das atitudes dos concorrentes (Amb1), disponibilidade de } \\
\text { mão-de-obra (Amb2), competição por compra de insumos (Amb3), tecnologia } \\
\text { aplicada no setor (Amb4), restrições legais, políticas e econômicas do setor } \\
\text { (Amb5), gostos e preferências dos clientes (Amb6) }\end{array}$ \\
\hline
\end{tabular}

Quadro 1 - Variáveis pesquisadas agrupadas por fatores

Fonte: Dados da pesquisa. 
Os dados coletados foram analisados quantitativamente. Inicialmente foi aplicada análise fatorial, com o objetivo de confirmar se as variáveis analisadas se agrupariam de acordo com o previsto na literatura. Apenas a variável referente ao uso do comércio eletrônico (Tec2) por parte das empresas, não foi agrupada adequadamente - com as demais variáveis entendidas pela literatura como representativas de tecnologia -, sendo assim, esta variável foi excluída do cálculo da carga fatorial desenvolvido posteriormente. Esta carga fatorial representa a transformação das variáveis pesquisadas em um único fator que maximiza o poder de explicação do conjunto de variáveis, também conhecido como fator dominante. As variáveis referentes a grau de diversificação e grau de verticalização, por exemplo, transformam-se no fator estratégia, e assim também para os demais. $\mathrm{O}$ fator tamanho da empresa não foi incluído nesta etapa, pois é composto por apenas uma variável, mas devido à sua variação foi transformado em log para a aplicação da regressão logística.

Para a aplicação da análise fatorial, assim como das demais técnicas estatísticas empregadas nesta pesquisa, foi utilizado o software estatístico SPSS (Statistical Package for the Social Sciences), versão 21.0. A rotação dos fatores foi realizada por meio do método varimax e a estimação dos valores dos mesmos pelo método dos mínimos quadrados ponderados. Para verificar se os dados eram adequados à aplicação da análise fatorial aplicouse o teste de KMO (Kaiser-Meyer-Olkin - Measure of Sampling Adequacy) e o teste de esfericidade de Barllet, que identificam se as variáveis são suficientemente correlacionadas umas com as outras para produzir fatores representativos (Hair et al., 2009).

O teste KMO, de acordo com Hair et al. (2009), pode variar de 0 a 1, sendo que quanto mais perto de 1, melhor, uma vez que, neste caso, cada uma das variáveis pode ser predita sem erros, com base nas demais. Ainda de acordo com os autores, caso o resultado do teste seja inferior a 0,5 a aplicação da análise fatorial é inaceitável. O teste de esfericidade de Barllet, por sua vez, evidencia a existência de correlação significativa entre pelo menos algumas das variáveis, buscando rejeitar, a um nível de significância de 5\%, a hipótese de que a matriz de correlação é uma matriz identidade.

O teste KMO empregado para verificar a confiabilidade dos dados apresentou saída entre 0,8 e 0,9 para as variáveis ambiente, porte, tecnologia e estrutura, enquanto o fator estratégia apresentou resultado de 0,5 , sendo todos considerados, desta forma, adequados para o emprego do teste estatístico. O resultado do teste de esfericidade de Barllet foi igual a 0 , quando analisados os fatores ambiente, tecnologia e estrutura, e igual a 0,046 no fator estratégia. Como 
o nível de significância necessário para os dados serem considerados adequados é de 5\%, e todos os resultados foram inferiores a este percentual, a técnica pôde ser aplicada.

A aplicação da análise fatorial gerou um fator dominante que explica aproximadamente $55 \%$ da variância total das variáveis do ambiente, $60 \%$ da estratégia, $63 \%$ da tecnologia e $65 \%$ da descentralização. Todas as variáveis relacionadas a estes fatores contingenciais mostraramse significativas na construção do fator dominante, uma vez que apresentaram cargas fatoriais acima de 0,6. Essa carga fatorial demonstra o coeficiente de correlação entre as variáveis pesquisadas e o fator gerado, e está evidenciada na Tabela 1.

\section{Tabela 1}

Análise fatorial: transformação das variáveis no fator dominante

\begin{tabular}{|c|c|c|c|c|c|}
\hline Fator & Variáveis & $\begin{array}{c}\text { Carga } \\
\text { fatorial }\end{array}$ & Fator & Variáveis & $\begin{array}{c}\text { Carga } \\
\text { fatorial }\end{array}$ \\
\hline \multirow{6}{*}{ Descentralização } & Desc1 & 0,830 & \multirow{6}{*}{$\begin{array}{c}\text { Estabilidade } \\
\text { ambiental }\end{array}$} & Amb1 & 0,769 \\
\hline & Desc2 & 0,645 & & Amb2 & 0,722 \\
\hline & Desc3 & 0,852 & & Amb3 & 0,702 \\
\hline & Desc4 & 0,887 & & Amb4 & 0,814 \\
\hline & Desc5 & 0,889 & & Amb5 & 0,670 \\
\hline & Desc6 & 0,739 & & Amb6 & 0,777 \\
\hline \multirow{5}{*}{ Tecnologia } & Tec1 & 0,677 & \multirow{2}{*}{ Estratégia } & Estra1 & 0,778 \\
\hline & Tec3 & 0,779 & & Estra2 & 0,778 \\
\hline & Tec4 & 0,804 & \multirow{3}{*}{\multicolumn{3}{|c|}{-}} \\
\hline & Tec5 & 0,864 & & & \\
\hline & Tec6 & 0,835 & & & \\
\hline
\end{tabular}

Fonte: Dados da pesquisa.

Diante da confiabilidade dos dados confirmada pelos testes de KMO e Barllet e do fator determinante gerado para o conjunto de variáveis de cada fator contingencial, que não apresentaram colinearidade, tornou-se possível a aplicação da regressão logística binária para verificar as relações entre os fatores estudados e a adoção do preço de transferência pelas empresas.

\section{Análise dos resultados}

\subsection{Descrição dos resultados}

As empresas selecionadas para esta pesquisa estão listadas no Catálogo de Exportadores Brasileiros, disponibilizado pela Confederação Nacional de Indústrias (CNI) no Portal de Indústrias Brasileiras. Das 90 empresas participantes $76 \%$ são enquadradas como indústria de transformação. Dentre essas, prevaleceram as fabricantes de máquinas e equipamentos (25\%). 
Na Tabela 2 é apresentada a estatística descritiva referente as variáveis: ambiente, estratégia, estrutura, tecnologia e porte

Tabela 2

Estatística descritiva

\begin{tabular}{|l|c|c|c|}
\hline \multicolumn{1}{|c|}{ Fator } & Média & Mediana & Desvio Padrão \\
\hline Ambiente & 5,95 & 6,17 & 1,94 \\
\hline Estratégia (diversificação) & 3,10 & 2,50 & 2,92 \\
\hline Estratégia (verticalização) & 5,58 & 5,00 & 3,03 \\
\hline Estrutura & 4,55 & 4,92 & 2,90 \\
\hline Tecnologia & 6,70 & 7,00 & 1,80 \\
\hline Porte & $\mathrm{R} \$ 86.550 .661,76$ & $\mathrm{R} \$ 45.500 .000,00$ & $\mathrm{R} \$ 106.188 .506,46$ \\
\hline
\end{tabular}

Fonte: Dados da pesquisa.

O ambiente das empresas pesquisados mostra-se moderado, com uma média de 5,95 e mediana de 6,17, de forma similar as empresas apresentam uma estrutura intermediária com média e mediana próximas a 5. No que se refere a estratégia, as organizações da amostra apresentaram baixo nível de diversificação e nível intermediário no que se refere a integração vertical. O fator com maiores médias foi o de tecnologia, apontando para o alto investimento das empresas exportadoras em automação. O porte das empresas pesquisadas teve grande variação, como pode ser identificado a partir do desvio padrão superior a 100 mil reais. Devido a variação apresentada empregou-se o logaritmo de porte na análise dos dados.

Dentre as empresas respondentes da pesquisa, 20 (22\%) informaram utilizar preço de transferência como instrumento de gestão. Os métodos empregados pelas empresas em suas transferências gerenciais estão evidenciados na Tabela 3.

Tabela 3

Métodos de preço de transferência gerencial usados nas empresas estudadas

\begin{tabular}{l|c|c}
\hline \multicolumn{1}{c|}{ Método de preço de transferência gerencial } & Frequência & $\mathbf{\%}$ \\
\hline Baseado no custo & $\mathbf{1 7}$ & $\mathbf{8 5 \%}$ \\
\hline Custo total & 8 & $50 \%$ \\
\hline Custo padrão & 5 & $25 \%$ \\
\hline Custo total mais margem de lucro & 3 & $19 \%$ \\
\hline Custo variável & 1 & $6 \%$ \\
\hline Baseado no mercado & $\mathbf{2}$ & $\mathbf{1 0 \%}$ \\
\hline Negociado entre gerentes & $\mathbf{1}$ & $\mathbf{5 \%}$ \\
\hline
\end{tabular}

Fonte: Dados da pesquisa.

Das empresas que afirmam empregar preço de transferência para avaliação de desempenho, 9 utilizam métodos que não permitem tal avaliação (custo total e custo variável). O preço de transferência baseado no custo total, predominante entre as empresas pesquisadas (8 empresas) transfere o item de uma divisão para outra com base no seu custo total real. Usando 
essa informação, a unidade vendedora do item, transferindo pelo custo, terá receita igual ao custo e lucro igual a zero, o que faz com que não seja possível a avaliação do seu desempenho financeiro a partir dessa informação. O preço de transferência baseado no custo variável tem as mesmas deficiências do método do custo total, acrescido o fato de que empregando essa abordagem a empresa transferirá apenas os custos variáveis de uma divisão para outra. O custo variável é útil para outras análises referentes a preço de transferência, que envolvem o impacto de decisões de transferência interna ou compra e venda no mercado, no resultado das unidades e da empresa como um todo. Além disso, se o custo real for usado para fazer as transferências, possíveis ineficiências existentes em divisões e que se refletem em maiores custos, serão aceitas e os custos envolvidos transferidos às unidades subsequentes.

O custo padrão (usado por 5 empresas) apesar de não transferir ineficiência entre as unidades, não faz uma distribuição adequada do lucro entre as unidades avaliadas, e da mesma forma o método baseado no custo total mais margem de lucro (usado por 3 empresas), que apesar de tentar distribuir o lucro entre as unidades avaliadas, também transfere possíveis ineficiências no preço de transferência e não tem a capacidade de simular situações de concorrência normal de mercado entre as divisões. Por outro lado, métodos que mais adequadamente permitem a avaliação de desempenho, como o método baseado no mercado e o negociado, são utilizados por apenas $15 \%$ da amostra.

Os resultados obtidos nesta pesquisa, desta forma, mostram-se contraditórios aos de estudos anteriores, como Borkowski (1992; 1997), Soutes (2006) e Grunow et al. (2010), uma vez que aponta para uma grande variação no uso, entre os métodos baseados no custo e o método baseado no mercado, enquanto os estudos anteriores apontavam certa similaridade no percentual de empresas que utilizavam tais métodos.

Nas empresas da amostra desta pesquisa, 4 das que apontaram métodos baseados nos custos como o principal utilizado na empresa, afirmaram utilizar algum método adicional. Duas informaram que o preço negociado também é utilizado, uma afirmou praticar adicionalmente o preço de mercado ajustado e a última afirmou alternar entre o custo total e o custo variável.

Destaca-se que estes achados, que demonstram alta aplicação do preço baseado nos custos e baixa utilização dos demais métodos, podem ser justificados pela simplicidade de obtenção de dados nos métodos baseados em custos, quando comparados, principalmente, ao preço de mercado, e pelo menor dispêndio de recursos quando analisados de forma comparativa ao método negociado, como destacado por Grunow et al. (2010). 
A utilização de outros métodos, como o administrado, dual price, ou modelos matemáticos para o cálculo do preço de transferência pelas empresas, não foi identificado, o que comprova a baixa utilização destes métodos em empresas brasileiras, como já identificado no estudo anterior de Grunow et al. (2010).

Na sequência aplicou-se a regressão logística sobre os dados das empresas estudadas, considerando como variável dependente a utilização, ou não, de preço de transferência, e como variáveis independentes os fatores gerados pela análise fatorial, representativos de descentralização, tecnologia, estratégia e ambiente. A variável independente foi incluída por meio de $\log$. O modelo de regressão logística está evidenciado na Tabela 4.

Tabela 4

Modelo de Regressão Logística

\begin{tabular}{lrrrr}
\hline \multicolumn{1}{c}{ Variáveis } & Coeficiente & \multicolumn{1}{c}{$\operatorname{Exp(B)}$} & \multicolumn{1}{c}{ Sig. } & Hipóteses \\
\hline Constant & $-5,515$ &, 004 &, 106 & \\
\hline Fator_Descentralização &, 400 & 1,492 &, 180 & H1 \\
\hline Fator_Tecnologia &, 365 & 1,441 &, 365 & H2 \\
\hline Fator_Estratégia &, 004 & 1,004 &, 990 & H3 \\
\hline Log_Tamanho &, 549 & 1,732 &, 214 & H4 \\
\hline Fator_Ambiente &, 172 & 1,187 &, 600 & H5 \\
\hline For & & & &
\end{tabular}

Fonte: Dados da pesquisa.

Ao analisar a coluna "Sig." da Tabela 4, nota-se que dentre os fatores listados, nenhum apresenta nível de significância (5\%) para a amostra estudada. Desta forma, com um nível de confiabilidade de $95 \%$ pode-se afirmar que os fatores descentralização, tecnologia, estratégia, tamanho e ambiente não são determinantes para o uso de preço de transferência, rejeitando todas as hipóteses da pesquisa.

\subsection{Discussão dos resultados}

A hipótese H1 supunha uma relação positiva entre o nível de descentralização das organizações e o uso de preço de transferência, a partir do pressuposto de que seu uso tende a ser uma consequência da descentralização e da necessidade de avaliar o desempenho das decisões tomadas pelos centros de responsabilidade. O fato da hipótese ser refutada neste estudo, contrariamente aos achados de Borkowski (1990, 1992), Holmstrom e Tirole (1991), que conseguiram inclusive estabelecer relação entre as empresas mais descentralizadas e a negociação do preço interno entre as divisões, aponta que as previsões da teoria a respeito da adoção de preço de transferência por empresas com esta estrutura não foram percebidas na prática das empresas brasileiras da amostra deste estudo. Isso indica que estas empresas podem 
não estar avaliando o desempenho financeiro de suas unidades, ou então utilizando outros indicadores - não financeiros - para avaliar desempenho.

A relação negativa entre o nível tecnológico das empresas e a adoção do preço de transferência $(\mathrm{H} 2)$, também não apresentou resultados estatisticamente significativos, o que evidencia que as empresas da amostra que apresentam alto nível tecnológico. Tal achado demostra que as empresas estudadas não seguem a teoria proposta por Adler (1996), que defende os riscos de implementação do preço de transferência para as empresas que necessitam investir em tecnologia, considerando que tais investimentos poderiam causar custos adicionais às divisões e alterar seus resultados de curto prazo, inibindo a vantagem competitiva da empresa. Tal posição das empresas pode estar diretamente relacionada aos achados da variável anterior: se há uma tendência das divisões das empresas descentralizadas não terem o desempenho avaliado pelo preço de transferência, não há motivo para os gestores temerem o aumento dos custos, ou seja, os investimentos em tecnologia não são inibidos. Outra observação é que, se os métodos de preço de transferência predominantes são baseados em custos, logo o desempenho das divisões não é avaliado pelo lucro.

Estes achados não necessariamente contrariam o resultado da pesquisa de Dikolli e Vaysman (2006), que encontraram evidências de variação dos métodos empregados pelas empresas de diferentes níveis de tecnologia, pois o estudo dos autores demonstrava que, tanto empresas com baixo nível tecnológico quanto empresas de alto nível tecnológico, empregavam preço de transferência. Como neste estudo um percentual muito alto de empresas afirmou empregar métodos baseados nos custos para definir o preço de transferência, não foi possível comparar os resultados de nível tecnológico com método, como feito por Dikolli e Vaysman (2006).

Embora Eccles (1983), Spicer (1988) e Adler (1996) tenham destacado a estratégia da empresa, em termos de integração vertical e diversificação, como variável determinante para a definição do preço de transferência, em que apenas empresas com níveis baixos de ambas não deveriam utilizar o preço de transferência, não foi encontrada relação positiva entre este fator e o uso de preço de transferência nesta pesquisa, rejeitando H3. Neste sentido, a presente pesquisa corrobora com os achados de Borkowski (1990) e Kouser et al. (2012), que não encontraram evidências de relação entre o preço de transferência e o nível de integração vertical e diversificação, respectivamente.

Quanto ao tamanho das empresas, também não é possível afirmar que há alguma tendência de empresas maiores utilizarem mais o preço de transferência do que empresas 
menores, contrariando os achados de Borkowski $(1990,1996)$ que apontavam relação entre as variáveis, sendo que empresas maiores tenderiam a usar um preço de transferência baseado no mercado, rejeitando assim a quarta hipótese (H4) deste trabalho. Kouser et al. (2012), por outro lado, que investigaram empresas num contexto similar ao brasileiro (situadas em um país em desenvolvimento), encontraram resultados similares aos deste estudo.

Em relação à variável tamanho, três amostras brasileiras já foram estudadas: empresas brasileiras que haviam sido indicadas para o Prêmio Transparência ou estavam na relação de Maiores e Melhores empresas de 2005 (Soutes, 2006), empresas listadas entre as 200 maiores do Espírito Santo (Gonzaga et al., 2010) e empresas exportadoras listadas no catálogo da CNI (a presente pesquisa), não havendo consenso sobre os resultados. Enquanto os achados de Gonzaga et al. (2010) apontaram que as chances de uma empresa com maiores ativos (considerando-se um desvio padrão) figurar como usuária de preço de transferência é de aproximadamente $15 \%$, em Soutes (2006).

Por fim, a H5 também foi rejeitada, já que os resultados para esta amostra contrariam os estudos de Borkowski (1990, 1992), que evidenciavam relação entre a estabilidade do ambiente em que a empresa está situada os métodos de precificação interna, demonstrando que os pressupostos de Donaldson (1999) sobre a influência do ambiente no contexto de tomada de decisões das empresas não se aplica ao contexto das empresas da amostra desta pesquisa, ao menos no que tange ao preço de transferência.

Assim, os resultados apontam que nenhuma das variáveis estudadas possui relação significativa com o uso ou não de preço de transferência, indo ao encontro da pesquisa desenvolvida por Kouser et al. (2012), que não encontrou evidências da relação entre fatores ambientais e organizacionais e o preço de transferência. Em comum os estudos têm a aplicação em empresas situadas em países não desenvolvidos, diferentes de outros estudos, realizados em países desenvolvidos, o que pode fornecer indícios de que os fatores que influenciam a adoção de preço de transferência, assim como de outros instrumentos gerenciais, podem variar de acordo com o contexto cultural e cognitivo dos países.

As características investigadas pela literatura como determinantes para opção pelo método de preço de transferência pelas empresas não têm relação com o uso do instrumento gerencial nas empresas brasileiras da amostra, sendo assim, recomenda-se que em países como o Brasil, as pesquisas em preço de transferência devam primeiramente aprofundar-se sobre os fatores que levam as empresas a utilizar preço de transferência e também outros instrumentos 
gerenciais, e identificar se há conhecimento por parte dos gestores e profissionais da área de controladoria acerca desses instrumentos e de sua aplicação para, então, avançar em pesquisas que visem compreender os fatores que são determinantes para a escolha entre um ou outro método de precificação interna, o melhor método a ser empregado de acordo com as características das empresas, e os efeitos desses métodos sobre divisões, gestores e empresas como um todo.

A possibilidade de desconhecimento do instrumento pelos profissionais que atuam nas organizações também leva ao questionamento da frequência com que o tema, preço de transferência, é ministrado em cursos de graduação em contabilidade no Brasil. Marques et al. (2016), analisando ementas de disciplinas de 46 universidades dos três estados do sul do Brasil, para avaliar os instrumentos de contabilidade gerencial que faziam parte das ementas de disciplinas, identificaram que preço de transferência constou em ementas de apenas 7 cursos investigados, representando 15,2\% da amostra. Esse também pode ser um dos motivos pelos quais poucas empresas adotam preço de transferência na avaliação de desempenho das áreas de responsabilidade, e isso leva a uma reflexão sobre a própria formação dos contadores que atuam nessas organizações, muitos dos quais, por desconhecerem esse instrumento também não têm compreensão dos benefícios que poderia trazer na avaliação de desempenho financeira de unidades descentralizadas.

Destaca-se ainda, que a amostra selecionada neste estudo contempla características particulares, pois são organizações que realizam transações internacionais, o que pode estar influenciando na determinação das escolhas gerenciais, levando essas empresas a simplificar suas transferências, não empregando instrumentos de avaliação de desempenho financeiro de divisões, ou usando metodologias mais simplificadas, como é o caso do preço baseado nos custos.

\section{Conclusões}

O objetivo desta pesquisa foi verificar os fatores que levam empresas exportadoras brasileiras a utilizar preço de transferência com abordagem gerencial, para avaliação de desempenho financeiro de divisões. Assim, foram propostas cinco hipóteses para investigar a relação entre os fatores contingenciais - descentralização, tecnologia, estratégia, tamanho da empresa e estabilidade do ambiente - e o uso de preço de transferência. Os dados que 
embasaram o estudo foram coletados junto a 90 empresas exportadoras brasileiras e analisados por meio de análise fatorial e de regressão logística.

Os resultados não permitiram a confirmação das hipóteses de pesquisa propostas, evidenciando que no contexto brasileiro tais fatores não se mostram como determinantes para a adoção desta ferramenta gerencial, corroborando com os achados da pesquisa de Kouser et al. (2012), aplicada a empresas localizadas no Paquistão. Estes achados podem estar relacionados a diferentes fatores como a característica das empresas estudadas (empresas exportadoras), a preferência da alta gestão e da equipe gerencial das empresas pelo uso de indicadores não financeiros para a avaliação de desempenho das unidades ou ainda a falta de conhecimento dos profissionais contábeis das organizações sobre a aplicação do preço de transferência como ferramenta gerencial.

Recomenda-se que pesquisas futuras busquem aprofundar o entendimento acerca do conhecimento dos profissionais contábeis, que atuam em empresas exportadoras, sobre o uso do preço de transferência para fins gerencial. Outra agenda relevante refere-se ao desenvolvimento de estudos qualitativos que busquem analisar a percepção da alta gestão e dos gerentes de nível médio acerca da utilidade deste instrumento gerencial.

\section{Referências}

Adams, L., \& Drtina, R. (2008). Transfer pricing for aligning divisional and corporate decisions. Business Horizons, 51(5), 411-417.

Adler, R. W. (1996). Transfer pricing for world-class manufacturing. Long Range Planning, 29(1), 69-75.

Alles, M., \& Datar, S. (1998). Strategic transfer pricing. Management Science, 44(4), 451-461.

Arya, A., \& Mittendorf, B. (2007). Interacting supply chain distortions: The pricing of internal transfers and external procurement. The Accounting Review, 82(3), 551-580.

Bailey, C. D., \& Collins, D. (2005). Goliath Corporation: An instructional case in transfer pricing policy. Journal of Accounting Education, 23(4), 264-276.

Baldenius, T., Reichelstein, S., \& Sahay, S. A. (1999). Negotiated versus cost-based transfer pricing. Review of Accounting Studies, 4(2), 67-91.

Banker, R. D., \& Datar, S. M. (1992). Optimal transfer pricing under postcontract information. Contemporary Accounting Research, 8(2), 329-352.

Bhattacharjee, S., \& Moreno, K. K. (2016). The Role of Informal Controls and a Bargaining Opponent's Emotions on Transfer Pricing Judgments. Contemporary Accounting Research.

Borkowski, Susan C. (1990) Environmental and organizational factors affecting transfer pricing: a survey. Journal of Management Accounting Research, 2, 78-99. 
(1992). Choosing a transfer pricing method: a study of the domestic and international decision-making process. Journal of international accounting, auditing and taxation, 1(1), 33-49.

(1996). An analysis (meta-and otherwise) of multinational transfer pricing research. The International Journal of Accounting, 31(1), 39-53.

(1997). Factors affecting transfer pricing and income shifting (?) between Canadian and US transnational corporations. The International Journal of Accounting, 32(4), 391-415.

Chang, L., Cheng, M., \& Trotman, K. T. (2008). The effect of framing and negotiation partner's objective on judgments about negotiated transfer prices. Accounting, Organizations and Society, 33(7), 704-717.

Chan, K. H., \& Lo, A. (2004). The influence of management perception of environmental variables on the choice of international transfer-pricing methods. The International Journal of Accounting, v. 39(1), 93-110.

Cheng, M. M., \& Hsieh, C. (2009). Transfer Price Negotiation in the Presence of Unequal Bargaining Power: The Effect of a Peer Evaluation Scheme on Inter-divisional Profit Distribution. Australian Accounting Review, 19(3), 195-206.

Chenhall, R. H. (2003). Management control systems design within its organizational context: findings from contingency-based research and directions for the future. Accounting, Organizations and Society, 28, 127-168.

Cools, M., Emmanuel, C., \& Jorissen, A. (2008). Management control in the transfer pricing tax compliant multinational enterprise. Accounting, Organizations and Society, 33(6), 603628.

Dikolli, S. S., \& Vaysman, I. (2006). Information technology, organizational design, and transfer pricing. Journal of Accounting and Economics, 41(1), 201-234.

Donaldson, L. (2001). The contingency theory of organizations. Handbook de estudos organizacionais, 1, 105-133.

Dorestani, A. (2004). Transfer price and equilibrium in multidivisional firms: an examination of divisional autonomy and central control. Applied Economics, 36(17), 1899-1906.

Eccles, R. G. (1985). The transfer pricing problem: A theory for practice.

Eccles, R. G. (1983). Control with fairness in transfer pricing. Harvard Business Review, v. 61, n. 6, p. 149-161.

Enzer, H. (1975). The static theory of transfer pricing. Naval Research Logistics (NRL), 22(2), 375-389.

Flavell, R. B. (1977). Divisionalization and transfer pricing: A review. Omega, 5(5), 543-556.

R. H., Noreen, E. W., \& Brewer, P. C. (2003). Managerial accounting. New York: McGrawHill/Irwin.

Gavious, A. (1999). Transfer pricing under capacity constraints. Journal of Accounting, Auditing \& Finance, 14(1), 57-72.

Ghosh, D., \& Boldt, M. N. (2006). The effect of framing and compensation structure on seller's negotiated transfer price. Journal of Managerial Issues, 453-467. 
Gonzaga, R. P., Da Luz, A. T. M., Guimarães, T. N., \& Valerio Jr, V. B. (2010). Associação entre práticas de contabilidade gerencial e tamanho das empresas: um estudo empírico. In: IV Congresso da Associação Nacional Dos Programas De Pós-Graduação Em Ciências Contábeis.

Göx, R. F. (2000). Strategic transfer pricing, absorption costing, and observability. Management Accounting Research, 11(3), 327-348.

Grunow, A., Beuren, I. M., \& Hein, N. (2010). Métodos de preço de transferência interna utilizados nas maiores indústrias do Brasil. Revista Economia \& Gestão, 10(24), 74-102.

Hair, J. F., Black, William C., Babin, B. J., Anderson, R. E., \& Tatham, Ronald L. (2009). Análise multivariada de dados. Porto Alegre: Bookman.

Hirshleifer, J. (1956). On the economics of transfer pricing. The Journal of Business, 29(3), 172-184.

Holmstrom, B., \& Tirole, J. (1991). Transfer pricing and organizational form. Journal of Law, Economics, \& Organization, 7(2), 201-228.

Johnson, E., Johnson, N. B., \& Pfeiffer, T. (2016). Dual transfer pricing with internal and external trade. Review of Accounting Studies, 21(1), 140-164.

Kachelmeier, S. J., \& Towry, K. L. (2002). Negotiated transfer pricing: Is fairness easier said than done?. The Accounting Review, 77(3), 571-593.

Kouser, R.; Qureshi, S.; Hassan, M.; Udman, A. (2012). A Survey on Transfer Pricing Practices in Manufacturing Sector of Pakistan. European Journal of Social Sciences, 29(3), 387-396.

Liu, G., Zhang, J., \& Tang, W. (2015). Strategic transfer pricing in a marketing-operations interface with quality level and advertising dependent goodwill. Omega, 56, 1-15.

Mahenthiran, S., Greenberg, P. S., \& Greenberg, R. H. (1993). The impact of computermediated communication on the processes and outcomes of negotiated transfer pricing. Accounting, Management and Information Technologies, 3(4), 229-248.

McAulay, L., \& Tomkins, C. R. (1992). A review of the contemporary transfer pricing literature with recommendations for future research. British Journal of Management, 3(2), 101-122.

Mura, A., Emmanuel, C., \& Vallascas, F. (2013). Challenging the reliability of comparables under profit-based transfer pricing methods. Accounting and Business Research, 43(5), 483505.

Porter, M. E. (1996). Estratégia competitiva. Rio de Janeiro: Campus.

Ravenscroft, S. P., Haka, S. F., \& Chalos, P. (1993). Bargaining behavior in a transfer pricing experiment. Organizational Behavior and Human Decision Processes, 55(3), 414-443.

Ruiz-Aliseda, F. (2003). Precios de transferencia en Oligopolios. Spanish Journal of Finance and Accounting/Revista Española de Financiación y Contabilidad, 32(118), 871-911.

Shi, Y., Kwak, W., \& Lee, H. (1998). Optimal Trade-offs of Multiple Factors inTransfer Pricing Problems.

Shih, M. S. (1996). Optimal transfer pricing method and fixed cost allocation. Abacus, 32(2), 178-195. 
Soutes, D. O. (2006). Uma investigação do uso de artefatos da contabilidade gerencial por empresas brasileiras. Tese de Doutorado apresentada ao programa de Contabilidade da Universidade de São Paulo. 116f.

\& De Zen, M. D. C. (2005). Estágios evolutivos da contabilidade gerencial em empresas brasileiras. In V Congresso USP de Controladoria e Contabilidade.

Spicer, B. H. (1988). Towards an organizational theory of the transfer pricing process. Accounting, Organizations and Society, 13(3), 303-322.

Tisdell, C. A. (1989). Transfer pricing: technical and productivity change within the firm. Managerial and Decision Economics, 10(3), 253-256.

Vaysman, I. (1998). A model of negotiated transfer pricing. Journal of Accounting and Economics, 25(3), 349-384.

Wells, M. C. (1968). Profit centres, transfer prices and mysticism. Abacus, 4(2), 174-181.

Widener, S. K. (2004). An empirical investigation of the relation between the use of strategic human capital and the design of the management control system. Accounting, Organizations and Society, 29(3), 377-399.

Wolff, M. (2007). Market price-based transfer price systems. Empirical evidence for effectiveness and preconditions. Problems and Perspectives in Management, 5(2), 66. 